\title{
Traditional versus Percutaneous Transforaminal Endoforaminal Discectomy Effect on the Nervous System Function and Serum LEK, GFAP and PGE-2 in Patients with Senile Lumbar Spinal Stenosis
}

\author{
Hejun Chen ${ }^{1}$, Junlian $\mathrm{Xue}^{2}$ and Xinjiang Wu${ }^{1}$
}

\begin{abstract}
Objective: To compare the influence of percutaneous transforaminal endoscopic discectomy (PTED) and traditional operation on the nervous system function and the serum leu-enkephalin (LEK), glial fibrillary acidic protein (GFAP) and prostaglandin E-2 (PGE-2) in patients with senile lumbar spinal stenosis.

Study Design: Experimental study.

Place and Duration of Study: Department of Orthopedics Two, Xinjiang Changji Hui Autonomous Prefecture People's Hospital, Xinjiang, China, from March 2017 to March 2018.

Methodology: A total of 146 patients with senile lumbar spinal stenosis were randomly divided into control group and observation group, 73 in each group. Control group underwent traditional operation, while the observation group underwent PTED. General situation of operation, serum LEK, GFAP, PGE-2, American Spinal Injury Association (ASIA) score and Japanese Orthopaedic Association (JOA) score were compared.

Results: Intraoperative blood loss in observation group was less than that in control group $(p<0.001)$. Both operation time and length of hospital stay in observation group were shorter than those in control group (both $p<0.001$ ). At 24 hours later after operation, both levels of serum LEK and ASIA score in observation group were higher than those in control group $(p=0.006$ and $p<0.001$, respectively), and levels of serum GFAP and PGE-2 and JOA score in observation group were all lower than those in control group (all $p<0.001$ ).

Conclusion: Compared with traditional operation, PTED has the advantages of less intraoperative blood loss, shorter operation time and length of hospital stay, etc. Besides, PTED can effectively reduce serum LEK, BFGF and PGE-2 expression in patients; and dramatically improve their nervous system function and lumbar function.
\end{abstract}

Key Words: Senile lumbar spinal stenosis, Percutaneous transforaminal endoscopic discectomy (PTED), Nervous system function.

\section{INTRODUCTION}

Lumbar spinal stenosis is a common disease in clinic, which can lead to lumbago and leg pain. It belongs to a nerve dysfunction caused by many factors, such as hypertrophied ligamentum flavum, hypertrophied facet joint, degenerative intervertebral disc, lumbar lateral recess caused by bulged and protruded intervertebral disc, etc.1,2 Lumbar spinal stenosis is a senile disease, and its main symptoms include lumbago, backache, intermittent claudication, cauda equina syndrome, etc., which can result in serious impacts on patients' quality of life, so surgery is required to treat it as early as

\footnotetext{
1 Department of Orthopedics Two, Xinjiang Changji Hui Autonomous Prefecture People's Hospital, Xinjiang, 831100-China

2 Department of Orthopedics, People's Hospital of Hutubi County, Xinjiang, 831100-China

Correspondence: Xinjiang Wu, Department of Orthopedics Two, Xinjiang Changji Hui Autonomous Prefecture People's Hospital, Xinjiang, 831100-China

E-mail:vjbjy2@163.com

Received: May 14, 2018; Accepted: November 03, 2018
}

possible.3,4 Formerly, traditional surgery is often adopted to treat senile lumbar spinal stenosis, but it has the disadvantages of severe trauma, long postoperative bed rest and slow recovery, which has been confirmed by clinical practice. 5,6 In recent years, the application of percutaneous transforaminal endoscopic discectomy (PTED) in patients with senile lumbar spinal stenosis has been gradually known for its advantages of less trauma and short time. 7,8 However, there are few studies on the influence of PTED on the nervous system function and the serum leu-enkephalin (LEK), glial fibrillary acidic protein (GFAP) and prostaglandin E-2 (PGE-2) of patients with senile lumbar spinal stenosis.

The objective of this study was to compare the influence of PTED and traditional operation on the nervous system function and the serum LEK, GFAP, and PGE-2 of patients with senile lumbar spinal stenosis.

\section{METHODOLOGY}

This study was conducted at the Department of Orthopedics Two, Xinjiang Changji Hui Autonomous Prefecture People's Hospital, Xinjiang, China, from March 2017 to March 2018. The study was approved by 
the Hospital Ethical and Research Committee. A total of 146 patients with senile lumbar spinal stenosis were selected as research objects. Patients with senile lumbar spinal stenosi were diagnosed CT or MRI imaging examination, family members explicitly abandoned open surgical treatment, and symptoms affecting daily life, were included. Those who had severe function abnormities in heart, lung, liver, kidney and blood coagulation, had severe ossification of posterior longitudinal ligament confirmed by imaging examination, and could not tolerate this surgery, were excluded. The patients were randomly divided into control group and observation group, with 73 in each group.

Traditional operation was used for the control group. With general anesthesia, the patients took a prone position. C-arm X-ray machine was used to locate. Taking lesion vertebrae as the center, the area with a length of about $11 \mathrm{~cm}$, was adopted to cut an incision through a median approach. Musculus sacrospinalis was peeled under the vertebral plate, and zygopophysis at the vertebral plate exposing the lesion node was also separated from spinous process. After the confirmation of screw length, direction and location, the pedicle screw was screwed in. The removal and decompression of vertebral plate and spinous process was carried out at the lesion node. The hypertrophied ligamentum flavum was removed, and the zygopophysis at two sides was kept. The neural plate and lateral recess were enlarged, and the fusion cage was installed. In addition, the incision was rinsed thoroughly. Meanwhile, the remaining bone cortexes, zygapophyseal joints and vertebral plates were fully implanted into the bone. The negative pressure drainage tube was placed in the incision, and the incision was closed layer by layer.

PTED was used for the observation group. With local anesthesia, the patients took a prone position. C-arm X-ray machine was used to locate. The central line between lumbar vertebrae and spinous process was localized via the superior border of intervertebral disc. The location at the horizontal distance of about $12.5 \mathrm{~cm}$ of $L_{4}$ vertebrae away from the midpoint was used as the puncture site between $L_{4}$ and $L_{5}$. Moreover, the location at the horizontal distance about $13.5 \mathrm{~cm}$ of the highest crista iliaca line away from the midpoint was used as the puncture site between $L_{1}$ and $L_{5}$. When puncture succeeded, $3 \mathrm{ml} 1 \%$ lidocaine was injected around the joints. The guidewire was inserted into epidural space along the puncture needle, and was served as the center to cut an incision with the length of about $7 \mathrm{~mm}$. The three-grade divergent tubes were successively inserted along the guide wire, and were pulled out after the confirmation of their position. The three-grade trepans were successively used to remove and polish. The intervertebral foramen was enlarged and the protective casing with the diameter of $7.5 \mathrm{~mm}$ was placed. After the confirmation of the position of casing, the intervertebral endoscope was placed to examine canalis spinalis. The protruded nucleus pulposus was removed under the endoscope, and the remnant nucleus pulposus was removed by radiofrequency ablation. Then, nerve root canal and intradiscal electrothermal annuloplasty was performed. If the patients had stenosis of lateral recess and intervertebral foramen, part of hypertrophied ligamentum flavum and superior articular process was removed. After dural sacs beat autonomously and nerve roots became loose, the protective casing was removed and the incision was sewed up in proper order.

The general situation of operation, including operation time, intraoperative blood, loss and length of hospital stay, were compared between the two groups. The levels of serum LEK, GFAP and PGE-2 of patients in the two groups was detected by enzyme-linked immunosorbent assay and compared at preoperatively and 24 hours postoperative.

The nerve function and the JOA score for the improvement of lumbar vertebra of patients in the two groups were recorded and evaluated by a neuro/spinal surgeon 24 hours postoperatively. ASIA scoring scale was used to evaluate the nerve function. There were 5 grades (1-5 scores) according to the degree of nerve function deficit. The higher the score, the less the nerve function deficit was. The JOA score for the improvement of lumbar vertebra included subjective symptom (9 scores), clinical sign (6 scores), limitation of daily activities (14 scores), and bladder function (-6-0 scores). The highest score was 29 and the lowest 0 . The higher the score, the more severe the dysfunction was.

Data was analysed in SPSS version 22. Mean value $\pm S D$ was calculated for numerical variables like intraoperative blood loss, operation time, length of hospital stay, serum LEK, BFGF and PGE-2, and examined by independent sample t-test. Frequencies and percentages were calculated for categorical variables like gender, and underlying diseases. The p-value $<0.05$ was considered statistically significant.

\section{RESULTS}

Among the 146 subjects, there were 80 (54.79\%) males and 66 (45.21\%) females. They were aged from 64 to 81 years, with an average age of $70.47 \pm 2.16$ years. The course of their illness was between 1 to 14 years, with the average time of $6.02 \pm 1.05$ years. Coexistent diseases included 90 (61.64\%) patients with hyper-

Table I: Comparison of the general situation of operation between two groups.

\begin{tabular}{l|c|c|c}
\hline Indices & $\begin{array}{c}\text { Control group } \\
(\mathrm{n}=73)\end{array}$ & $\begin{array}{c}\text { Observation group } \\
(\mathrm{n}=73)\end{array}$ & p-value \\
\hline Operation time $(\mathrm{min})$ & $92.78 \pm 15.81$ & $63.16 \pm 11.09$ & $<0.001$ \\
\hline $\begin{array}{l}\text { Intraoperative blood } \\
\text { loss }(\mathrm{mL})\end{array}$ & $196.35 \pm 52.12$ & $108.91 \pm 17.32$ & $<0.001$ \\
\hline Length of hospital stay $(\mathrm{d})$ & $9.42 \pm 1.74$ & $4.15 \pm 0.48$ & $<0.001$ \\
\hline
\end{tabular}


Table II: Comparison of the level of serum LEK, GFAP and PGE-2 between the two groups.

\begin{tabular}{|c|c|c|c|c|}
\hline Indices & Time & Control group $(n=73)$ & Observation group $(n=73)$ & $p$-value \\
\hline \multirow[t]{2}{*}{ LEK $(p g / m L)$} & Pre-operative & $86.94 \pm 2.75$ & $86.58 \pm 3.12$ & 0.461 \\
\hline & At 24 hours later after operation & $107.86 \pm 15.81$ & $116.41 \pm 21.08$ & 0.006 \\
\hline \multirow[t]{2}{*}{ GFAP $(\mu \mathrm{g} / \mathrm{L})$} & Pre-operative & $2.03 \pm 0.58$ & $2.04 \pm 0.61$ & 0.919 \\
\hline & At 24 hours later after operation & $1.69 \pm 0.40$ & $1.03 \pm 0.20$ & $<0.001$ \\
\hline \multirow[t]{2}{*}{ PGE-2 (pg/mL) } & Pre-operative & $57.03 \pm 4.11$ & $57.15 \pm 4.46$ & 0.866 \\
\hline & At 24 hours later after operation & $40.55 \pm 6.01$ & $29.72 \pm 1.58$ & $<0.001$ \\
\hline
\end{tabular}

Table III: Comparisons of the ASIA and JOA score between two groups at 24 hours later after operation.

\begin{tabular}{l|c|c|c}
\hline Indices & $\begin{array}{c}\text { Control group } \\
(\mathrm{n}=73)\end{array}$ & $\begin{array}{c}\text { Observation group } \\
(\mathrm{n}=73)\end{array}$ & $\mathrm{p}$-value \\
\hline $\mathrm{ASIA}$ (score) & $2.92 \pm 0.52$ & $4.64 \pm 1.06$ & $<0.001$ \\
$\mathrm{JOA}$ (score) & $16.06 \pm 2.21$ & $11.78 \pm 1.73$ & $<0.001$ \\
\hline
\end{tabular}

tension, $34(23.29 \%)$ patients with diabetes, and 22 $(15.07 \%)$ patients with coronary heart disease.

The intraoperative blood loss in the observation group was less than that in the control group $(p<0.001)$. The operation time and the length of hospital stay in the observation group were both shorter than that in the control group (both $\mathrm{p}<0.001$, Table I).

Preoperatively, there was no significant difference in serum LEK, GFAP and PGE-2 between two groups $(p=0.461,0.919$ and 0.866 , respectively). At 24 hours after operation, the level of serum LEK in the observation group was higher than that in the control group ( $p=0.006)$, and the levels of serum GFAP and PGE-2 in the observation group were lower than those in the control group (both $p<0.001$, Table II).

Twenty-four hours after operation, the ASIA score in the observation group was higher than that in the control group $(p<0.001)$, and the JOA score in the observation group was lower than that in the control group $(p<0.001$, Table III).

\section{DISCUSSION}

Surgery is the ultimate option to treat senile lumbar spinal stenosis in clinic.9,10 Previously, traditional surgery was often used to treat senile lumbar spinal stenosis, but there were some limitations in its effects. With the continuous improvement of people's living standard, patients with senile lumbar spinal stenosis gradually have a higher requirement to the surgery. Their demand cannot be satisfied only by relieving pain; thus it has gradually become the basic requirements in the surgical treatment of senile patients to recover the ability of daily life and enhance the quality of life. Over the past years, with the rapid development of minimally invasive and microsurgical techniques, PTED has been gradually recognized by clinicians, patients and their families; and was widely used in treatment of senile lumbar spinal stenosis. ${ }^{11}$

PTED was performed in this study to treat the patients with senile lumbar spinal stenosis. The results indicated that the intraoperative blood loss in the observation group was less than that in the control group, and the operation time as well as the length of hospital stay was shorter than that in the control group. These suggest that the application of PTED in treatment of senile lumbar spinal stenosis can significantly decrease intraoperative blood loss and shorten operation time as well as length of hospital stay. This conclusion is basically consistent with other studies. ${ }^{12}$ As temperature-controlled plasma radiofrequency ablation is applied in PTED, hemostatic treatment can be conducted timely and carefully in any surgical area and after any procedure, thus reducing intraoperative blood loss. Moreover, the incision is small in PTED, only $7 \mathrm{~mm}$, which effectively avoids cutting a big operative incision and damaging the structure of joints, bones and soft tissues. As endoscope is used during operation, the situation of proliferated bone tissue, nucleus pulposus and protruded nerve root is fully exposed in the surgical field, which helps remove easily, decrease nervous tissue adhesions postoperatively, and shorten operation time as well as length of hospital stay. 13

LEK is mainly distributed in hypothalamus, hippocampus, cerebral cortex, corpus striatum, amygdaloid nucleus, etc. It is an endogenous neuropeptide with the highest content in the brain and has an extensive analgesic effect. ${ }^{14,15}$ GFAP, the marker protein of astrocytes, can not only regulate cell metabolism, form and maintain the blood-brain barrier, produce and release neurotrophic factors, but also participate in the connection of cell nucleus and cell membrane, the cytoskeletal reorganization and myelinization, support and stabilize the neuronal structure, and play an important role in keeping the morphology and function of astrocytes. ${ }^{16,17}$ Relevant data shows that the level of serum GFAP is positively correlated with the severity of patients with craniocerebral injury. ${ }^{18}$ PGE-2, a marker factor of inflammatory reaction, can stimulate abnormal discharge of nerve roots, thus resulting in pain. ${ }^{19}$ After the level of PGE-2 rises, nerve roots will become more sensitive to pain-producing substances and lower the pain threshold to make the body feel pain. If blood vessels get damaged, prostaglandin can cause vasospasm. 20 In addition, PGE-2 can also induce the release of inflammatory factors and make nervous centralis more sensitive, thus increasing pain. ${ }^{21}$

The results of this study showed 24 hours later after operation, the level of serum LEK in the observation 
group was higher than that in the control group, and the levels of serum GFAP and PGE-2 in the observation group were lower than those in the control group; the ASIA score in the observation group was higher than that in the control group, and the JOA score in the observation group was lower than that in the control group. These suggest that PTED can relieve nerve root compression timely and effectively, and regulate the expression of serum LEK, GFAP and PGE-2 effectively, thus alleviating nerve function deficit and finally realizing the improvement of lumbar function.

Limitations of the study were as follow: Firstly, the sample was relatively small. Secondly, the observation time of clinical outcomes was relatively short. Further study need to expand the sample size and extend the observation time.

\section{CONCLUSION}

PTED is superior to traditional operation in treatment of senile lumbar spinal stenosis. It has a positive clinical effect in terms of regulating the expression of serum LEK, GFAP and PGE-2, improving nerve function and lumbar function, etc.; and so is suitable for popularization and application in the clinic treatment of senile lumbar spinal stenosis.

\section{REFERENCES}

1. van den Akker-van Marle ME, Moojen WA, Arts MP, VleggeertLankamp CL, Peul WC Leiden - The Hague Spine Intervention Prognostic Study Group. Interspinous process devices versus standard conventional surgical decompression for lumbar spinal stenosis: Cost utility analysis. Spine J 2016; 16:702-10.

2. Försth $P$, Ólafsson $G$, Carlsson $T$, Frost $A$, Borgström F, Fritzell $P$, et al. A randomized, controlled trial of fusion surgery for lumbar spinal stenosis. N Engl J Med 2016; 374:1413-23.

3. Ragab AA, Fye MA, Bohlman HH. Surgery of the lumbar spine for spinal stenosis in 118 patients 70 years of age or older. Spine (Phila Pa 1976) 2003; 28:348-53.

4. de Graaf I, Prak A, Bierma-Zeinstra S, Thomas S, Peul W, Koes B. Diagnosis of lumbar spinal stenosis: A systematic review of the accuracy of diagnostic tests. Spine (Phila Pa 1976) 2006; 31:1168-76.

5. Westermark P, Westermark GT, Suhr OB, Berg S. Transthyretin-derived amyloidosis: Probably a common cause of lumbar spinal stenosis. Ups J Med Sci 2014; 119: 223-8.

6. Ohtori S, Mannoji C, Orita S, Yamauchi K, Eguchi Y, Ochiai N, et al. Mini-open anterior retroperitoneal lumbar interbody fusion: Oblique lateral interbody fusion for degenerated lumbar spinal kyphoscoliosis. Asian Spine J 2015; 9:565-72.
7. Park $\mathrm{CH}$, Lee SH. Effectiveness of percutaneous transforaminal adhesiolysis in patients with lumbar neuroforaminal spinal stenosis. Pain Physician 2013; 16:E37-43.

8. Gadjradj PS, Harhangi BS. Percutaneous transforaminal endoscopic discectomy for lumbar disk herniation. Clin Spine Surg 2016; 29:368-71.

9. Herno A, Airaksinen O, Saari T, Luukkonen M. Lumbar spinal stenosis: A matched-pair study of operated and non-operated patients. Br J Neurosurg 1996; 10:461-5.

10. Gepstein R, Arinzon Z, Adunsky A, Folman Y. Decompression surgery for lumbar spinal stenosis in the elderly: Preoperative expectations and postoperative satisfaction. Spinal Cord 2006; 44:427-31.

11. Knaub MA, Won DS, Mcguire R, Herkowitz HN. Lumbar spinal stenosis: Indications for arthrodesis and spinal instrumentation. Instr Course Lect 2005; 54:313-9.

12. Wen B, Zhang $X$, Lin Z, Peng $H$, Zheng G. Percutaneous endoscopic transforaminal lumbar spinal canal decompression for lumbar spinal stenosis. Medicine (Baltimore) 2016; 95: e5186.

13. Ahn Y. Percutaneous endoscopic decompression for lumbar spinal stenosis. Expert Rev Med Devices 2014; 11:605-16.

14. Jiang CL, You ZD, Lu CL, Xu D, Wang AJ, Wang YX, et al. Leuenkephalin induced by IL-2 administration mediates analgesic effect of IL-2. Neuroreport 2000; 11:1483-5.

15. Guevremont R, Purves RW. High field asymmetric waveform ion mobility spectrometry-mass spectrometry: An investigation of leucine enkephalin ions produced by electrospray ionization. J Am Soc for Mass Spectrom 1999; 10:492-501.

16. Fraser DD, Close TE, Rose KL, Ward R, Mehl M, Farrell C, et al. Severe traumatic brain injury in children elevates glial fibrillary acidic protein in cerebrospinal fluid and serum. Pediatr Crit Care Med 2011; 12:319-24.

17. Wiesmann M, Steinmeier E, Magerkurth O, Linn J, Gottmann D, Missler U. Outcome prediction in traumatic brain injury: comparison of neurological status, CT findings, and blood levels of s100B and GFAP. Acta Neurol Scand 2010; 121:178-85.

18. Shehab HA, Nassar YH. Neuromarkers as diagnostic adjuvant to cranial CT in closed traumatic brain injury patients admitted to ICU: A preliminary comparative study. Egypt $J$ Anaesth 2010; 26:267-72.

19. Birrell GJ, Mcqueen DS. The effects of capsaicin, bradykinin, PGE2, and cicaprost on the discharge of articular sensory receptors in vitro. Brain Res 1993; 611:103-7.

20. Zimmermann K, Reeh PW, Averbeck B. ATP can enhance the proton-induced CGRP release through $\mathrm{P} 2 \mathrm{Y}$ receptors and secondary PGE2 release in isolated rat dura mater. Pain 2002; 97:259-65.

21. Johansson T, Narumiya S, Zeilhofer HU. Contribution of peripheral versus central EP1 prostaglandin receptors to inflammatory pain. Neurosci Lett 2011; 495:98-101. 